\title{
Modeling the Dynamics of Infectious Disease Under the Influence of Environmental Pollution
}

\author{
Nitu Kumari ${ }^{1}$ - Sandeep Sharma ${ }^{1}$
}

Published online: 26 April 2018

(C) Springer (India) Private Ltd., part of Springer Nature 2018

\begin{abstract}
Environmental pollution is one of the leading causes of mortality across the globe. There are evidences in literature which reflects the fact that regular exposure to environmental pollution leads to reduced immunity in human population. Therefore, we introduce environmental pollution as one of the concepts in understanding the dynamics of infectious disease. We propose a new SIS type epidemic model to study the impact of environmental pollution on the spread of infectious diseases. We divide the susceptible individuals into two compartments out of which one contains the pollution affected individuals. The present study demonstrates that we can not ignore environmental pollution during the study of a disease model. Till date, there are no studies which show the significance and impact of environmental pollution on the spread of infectious diseases. The expression of basic reproduction number is obtained for the proposed model. A detailed dynamical analysis of the model has been performed using the theory of ordinary differential equations, dynamical system and basic reproduction number. Numerical simulations along with sensitivity analysis are performed to support our analytical findings.
\end{abstract}

Keywords Infectious disease - Environmental pollution - Basic reproduction number · Backward bifurcation · Stability

Mathematics Subject Classification 92D30 - 34D20 - 34D23

Nitu Kumari

nitu@iitmandi.ac.in

Sandeep Sharma

sampark81@gmail.com

1 School of Basic Sciences, Indian Institute of Technology, Mandi, Mandi, Himachal Pradesh 175001, India 


\section{Introduction}

The increasing burden of infectious or communicable diseases is one of the major menace of the present time. Almost every country across the globe is suffering from different infectious diseases (e.g TB, AIDS, Influenza, Dengue, Malaria, SARS, Cholera, Influenza etc.). The occurrence of the infectious diseases result in huge loss in terms of life, money or both. Therefore, it is easy to conclude that mitigation of the spread of infectious diseases is an important problem of the present time. The agencies and organizations working on infectious diseases are trying to develop some effective control programs and measures to reduce the burden of infectious diseases. Different techniques, like vaccination, awareness, treatment etc., have been frequently used to control the spread of various infectious diseases. Despite all the efforts, the infectious diseases are the grievous part of modern society.

Mathematical modeling emerges as an effective tool to understand the dynamics of infectious diseases. Since the first compartmental model given by Kermack and Mckendrick [30], a number of mathematical models involving some complex assumptions are proposed. With the advent of theory of differential equations and dynamical systems, people are able to formulate some realistic mathematical models which provide concrete information about the spread of an infectious disease. These mathematical models enable us to explore the conditions to manage, reduce and eradicate the disease [3,9]. A variety of mathematical models exploring different dimensions have been proposed and analyzed in the literature. The availability of exhaustive literature does not allow to review and report all the approaches here. Nevertheless, we provide an overview of some of the important multidimensional applications of mathematical models in epidemiology in Table 1.

Despite this, there is no mathematical model available to study the role of environmental pollution in the spread of disease. However, some of the studies find that environmental fluctuations affect the behavior of the disease significantly. In particular, various studies investigate the impact of climate and environmental changes on vector-borne diseases $[6,28$, 55]. Some other studies draw general sketch of the interplay of environmental change and infectious disease [16,34,42,45].

Environmental pollution is a grievous truth of the present society. Environmental pollution, which includes air, water and soil pollution, is one of the leading causes of mortalities across the globe. The toxic chemicals present in the environment causes several hazardous effects on the living species. The impact of a pollutant on a living species depends on various factors including the type of pollutant, the degree of exposure, the individual health status and

Table 1 Different dimensions of mathematical modeling of epidemics

\begin{tabular}{lll}
\hline S. no. & Type & References \\
\hline 1. & $\begin{array}{l}\text { Models with different types of transmission } \\
\text { rates }\end{array}$ & {$[1,2,46]$ and the references cited therein } \\
2. & Models consider vaccination & {$[5,7,32]$ and references cited therein } \\
3. & Models with awareness & {$[15,23,49]$ and references cited therein } \\
4. & Models with seasonality & {$[41,44,56]$ and references cited therein } \\
5. & Treatment models & {$[18,54,57]$ and references cited therein } \\
6. & Models with delay & {$[38,51,53]$ and references cited therein } \\
7. & Models on vector borne diseases & {$[8,12,19]$ and references cited therein } \\
8. & Waterborne diseases models & {$[14,50,58]$ and references cited therein }
\end{tabular}


genetics. As different anthropogenic activities generate a variety of pollutants. Therefore, a number of studies investigate the impact of same on living species (see [17,24,25,29] and references cited therein). All the studies pointed out that increase in environmental pollution significantly reduce the chance of survival of population and biomass. Unfortunately, the factors which lead to the growth and development (industrialization, transportation etc.) of human race significantly increase the level of pollution. Due to the increasing population and demands of the society, we are in no position to control the events related to the development. The hazardous effects caused by environmental pollution allow us to conclude that environmental pollution is one of the major stress of the present time [35]. Further, Some studies highlight that regular exposure to pollution results in the disruption of immune system $[22,27,43,47]$. The smaller changes resulted from early exposure to chemical may increase susceptibility and the risk of disease significantly [22,31,35]. From this discussion, we can immediately conclude that neglecting the environmental pollution during disease modeling may result in false and vague predictions. In spite of this fact, the environmental pollution remains untouched by the multidimensional development of the mathematical modeling of infections diseases. To address this serious issue, we propose an SIS type epidemic model incorporating the environmental pollution (stress).

The manuscript is organized into nine sections. The mathematical model is given in section two. The equilibrium points and basic reproduction number are obtained in section three. The possibility of backward bifurcation is exhorted in section four. Section five contains the stability analysis of the equilibrium points. To demonstrate the impact of environmental pollution on the dynamics of disease, an extensive numerical solution is performed in section six. The sensitivity analysis of the basic reproduction number is carried out in section seven. Section eight contains the conclusion and epidemiological insight of the present study. The paper ends with a discussion on challenges and future scope of the proposed work in section nine.

\section{Mathematical Model}

Based on the literature survey on environmental pollution and infectious diseases, the following points can be highlighted:

1. Environmental pollution is a major environmental stress of the modern times.

2. The prenatal exposure to pollutants affects the newborns and infants.

3. The health of individuals exposed to environmental stress are compromised and they become more prone to infectious diseases.

4. The environmental stress also damages the immune system and makes the individual weak to resist various kinds of infections.

Motivated from these findings, we propose a compartmental model to investigate the impact of environmental pollution on the dynamics of the disease. Let us assume that human population under consideration is $N$. We divide $N$ into three mutually exclusive compartments $X, P$ and $Y$. Here, $X$ represents the susceptible population who are not affected by pollution, the pollution affected individuals, here after are referred as stressed individuals, represented by $P . Y$ represents the compartment of infected individuals. It is assumed that susceptible individuals acquired infection through direct contact with infected individuals. Some more pivot assumptions involve in the model formulation are given below 
(i) As the prenatal exposure to the environmental pollution has various adverse effects, therefore it is assumed that a fraction $m$ of total newborns will enter into $X$ class and the remaining fraction $(1-m)$ will enter into the $P$ class.

(ii) Individuals from $X$ class will enter into $P$ class at a constant rate $\theta$.

(iii) As the environmental pollution has various hazardous effects on stressed individuals including the weakening of immunity, hence it is also assumed that the transmission rate for pollution affected individuals $(P)$ is more as compared to unaffected individuals $(X)$.

Consequently the proposed deterministic nonlinear model can be formulated as under:

$$
\begin{aligned}
& \frac{d X}{d t}=m A-\theta X-\lambda X Y+n \xi Y-\mu X \\
& \frac{d P}{d t}=(1-m) A+\theta X-\lambda\left(1+\delta \lambda^{\prime}\right) P Y+(1-n) \xi Y-\mu P \\
& \frac{d Y}{d t}=\lambda X Y+\lambda\left(1+\delta \lambda^{\prime}\right) P Y-(\xi+\phi+\mu) Y
\end{aligned}
$$

In the model system (1)-(3), $A$ represents the recruitment rate of the newborns. The disease transmission rate for individuals of compartment $X$ is $\lambda$. Transmission rate for the individuals of $P$ class is $\lambda\left(1+\delta \lambda^{\prime}\right)$, where $\delta$ scales the amount by which environmental pollution affects the transmission rate and $\lambda^{\prime}$ gauge the effect of pollution $\lambda$. The biological reasoning behind stress related transmission rate can be find in [35]. $\theta$ is the rate at which the susceptible individuals are transmitted into the stressed compartment. The natural mortality rate is same for all individuals and is represented by $\mu$. The infected individuals are recovered at a constant rate $\xi$. A proportion $n$ of recovered individuals is transferred back to the susceptible compartment while the remaining proportion $(1-n)$ enters into the stressed compartment. The infected individuals also die due to disease related death rate $\phi$. The flow diagram of the proposed model system is given in Fig. 1.

All the parameters considered in the model are positive and the model is studied under the following initial conditions

$$
X(0) \geq 0, P(0) \geq 0 \text { and } Y(0) \geq 0
$$

The mathematical studies available in the literature have either addressed the hazardous impacts of environmental pollution on human population or studied the cases of epidemics. To the best of our knowledge, the mathematical model proposed in [33] is the only mathematical study available in the literature, where simultaneous impact of environmental pollution

Fig. 1 Flow diagram of the model

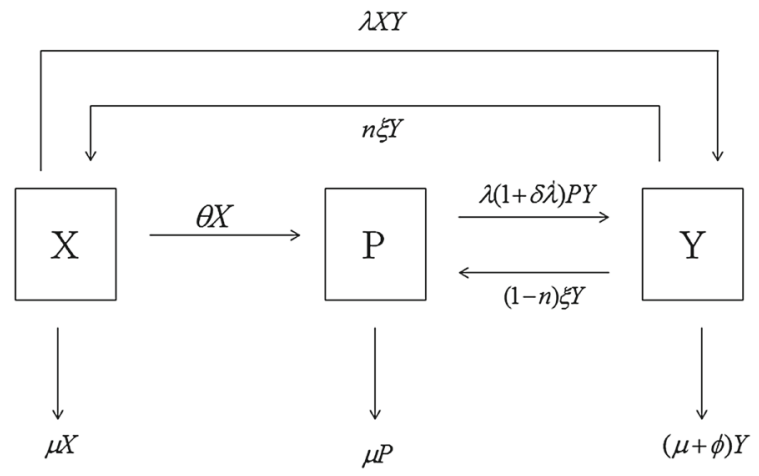


(caused by disinfectant by-products) and epidemics on human population is studied. The proposed model investigates the impact of environmental pollution in general on the dynamics of SIS type epidemic model.

\section{Existence of Equilibria and Basic Reproduction Number}

The region of attraction [20], for the purpose of dynamical study of our model system (1)-(3), is given by the following lemma

\section{Lemma 1 The set}

$$
\Omega=\left\{(X, P, Y): 0 \leq X, P, Y \leq \frac{A}{\mu}\right\}
$$

attracts all the solutions starting in the interior of positive orthant.

The model system possesses the following two equilibria

(i) The disease free equilibrium $E^{0}=\left(X^{0}, P^{0}, 0\right)$, where $X^{0}=\frac{m A}{(\theta+\mu)}$ and $P^{0}=$ $\frac{(1-m) A(\theta+\mu)+\theta m A}{\mu(\theta+\mu)}$.

(ii) The endemic equilibrium $E^{*}=\left(X^{*}, P^{*}, Y^{*}\right)$.

The existence of disease free equilibrium point is trivial. So, here we will study the existence of endemic equilibrium point $E^{*}$ in detail. Before the existence of $E^{*}$, we will first obtain the expression of basic reproduction number $R_{0}$. It helps us in measuring the number of secondary infections produced by a typical infected individual (assumed infectious) during his/her entire lifetime as infectious (infectious period) when introduced to a population of susceptibles [26,52]. The threshold parameter $R_{0}$ is useful in characterizing the spread of an infectious disease. Here, we use the next generation matrix method $[26,52]$ to obtain the expression of basic reproduction number. For our model the matrices $\mathscr{F}$ and $\mathscr{V}$ are given as:

$$
\begin{aligned}
& \mathscr{F}=\left[\begin{array}{c}
\lambda X Y+\lambda\left(1+\delta \lambda^{\prime}\right) P Y \\
0 \\
0
\end{array}\right] \\
& \mathscr{V}=\left[\begin{array}{c}
(\xi+\phi+\mu) Y \\
-(1-m) A-\theta X+\lambda\left(1+\delta \lambda^{\prime}\right) P Y-(1-n) \xi Y+\mu X_{2} \\
-m A+\theta X+\lambda X Y-n \xi Y+\mu X
\end{array}\right]
\end{aligned}
$$

Let $\mathrm{F}$ be the Jacobian of $\mathscr{F}$ at disease free equilibrium point

$$
F=\left[\lambda X^{0}+\lambda\left(1+\delta \lambda^{\prime}\right) P^{0}\right]
$$

Similarly, $\mathrm{V}$ is the Jacobian of $\mathscr{V}$, which takes the form

$$
V=[(\xi+\phi+\mu)]
$$

Now, the basic reproduction number $R_{0}$ for the model is the spectral radius of $\left(F V^{-1}\right)$, which is

$$
R_{0}=\frac{\lambda m A \mu+\lambda\left(1+\delta \lambda^{\prime}\right) A\{(\theta+\mu)-\mu m\}}{\mu(\theta+\mu)(\xi+\phi+\mu)}
$$


Next, the solution of following set of algebraic equations gives the endemic equilibrium point $E^{*}=\left(X^{*}, P^{*}, Y^{*}\right)$ for the proposed model system:

$$
\begin{aligned}
& m A-\theta X-\lambda X Y+n \xi Y-\mu X=0 \\
& (1-m) A+\theta X-\lambda\left(1+\delta \lambda^{\prime}\right) P Y+(1-n) \xi Y-\mu P=0 \\
& \lambda X Y+\lambda\left(1+\delta \lambda^{\prime}\right) P Y-(\xi+\phi+\mu) Y=0
\end{aligned}
$$

From Eq. (4), we obtain $X^{*}=\frac{m A+n \xi Y}{(\lambda Y+(\theta+\mu))}$

Similarly, from Eq. (5), we get $P^{*}=\frac{(1-m) A+\theta X^{*}+(1-n) \xi Y}{\lambda\left(1+\delta \lambda^{\prime}\right) Y+\mu}$

Using $X^{*}$ and $P^{*}$ in Eq. (6), we obtain a quadratic equation in $Y$ as

$$
F(Y)=A_{1} Y^{2}+A_{2} Y+A_{3}=0
$$

where,

$$
\begin{aligned}
A_{1}= & -\lambda^{2}\left(1+\delta \lambda^{\prime}\right)(\phi+\mu), \\
A_{2}= & -\left[(\xi+\phi+\mu)\left(\lambda\left(1+\delta \lambda^{\prime}\right)(\theta+\mu)+\lambda \mu\right)-\lambda n \mu \xi-\lambda^{2}\left(1+\delta \lambda^{\prime}\right) A\right. \\
& \left.\quad-\lambda\left(1+\delta \lambda^{\prime}\right) n \xi \theta-\lambda\left(1+\delta \lambda^{\prime}\right)(1-n) \xi(\theta+\mu)\right] \\
A_{3}= & \mu(\xi+\phi+\mu)(\theta+\mu)\left(R_{0}-1\right)
\end{aligned}
$$

It is evident from the expressions of $A_{1}, A_{2}$ and $A_{3}$ that $A_{1}$ is always negative. Now it is clear, by Descartes's rule of sign, that Eq. (7) always has a unique positive root whenever $R_{0}>1$. Moreover, Eq. (7) may have more than one positive roots if $R_{0}<1$ and $A_{2}>0$. This will results in more complicated dynamics. In particular, the model system (1)-(3) may undergo backward bifurcation if $R_{0}<1$. The existence of backward bifurcation violates the classical epidemiological requirement of disease eradication. In this case, more efforts are required to eradicate the disease. In the next section, we perform a detailed analysis to investigate the existence of backward bifurcation.

\section{Existence of Backward Bifurcation}

In this section, we will explore the possibility of backward bifurcation. To achieve this task, we calculate the bifurcation coefficients using center manifold theory [11]. From the expression of $R_{0}$ it is clear that it is directly related to $\lambda$. Therefore, we select $\lambda$ as the bifurcation parameter. Moreover, $R_{0}=1$ implies that $\lambda^{*}=\frac{\mu(\theta+\mu)(\xi+\phi+\mu)}{m A \mu+\left(1+\delta \lambda^{\prime}\right) A\{(\theta+\mu)-\mu m\}}$. Further, the Jacobian matrix of the model system (1)-(3) around the disease free equilibrium is

$$
J_{0}=\left[\begin{array}{ccc}
-(\theta+\mu) & 0 & -\lambda X^{0}+n \xi \\
\theta & -\mu & -\lambda\left(1+\delta \lambda^{\prime}\right) P^{0}+(1-n) \xi \\
0 & 0 & \lambda X^{0}+\lambda\left(1+\delta \lambda^{\prime}\right) P^{0}-(\xi+\phi+\mu)
\end{array}\right]
$$

At $\lambda=\lambda^{*}, J_{0}$ has a simple zero eigenvalue while other eigenvalues are negative. The right eigenvectors, $\left(w_{1}, w_{2}, w_{3}\right)$ of $J_{0}$ at $\lambda=\lambda^{*}$ can be obtained as under 


$$
\begin{aligned}
& w_{1}=\frac{1}{(\theta+\mu)}\left[\frac{-m \mu(\xi+\phi+\mu)+n \xi\left\{m \mu+\left(1+\delta \lambda^{\prime}\right)(\theta+\mu)-\mu m\right\}}{\left\{m \mu+\left(1+\delta \lambda^{\prime}\right)((\theta+\mu)-\mu m)\right\}}\right] \\
& w_{2}=\frac{1}{\mu}\left[\theta w_{1}+\frac{-(\xi+\phi+\mu)\left(1+\delta \lambda^{\prime}\right)((\theta+\mu)-\mu m)+(1-n)\left\{\mu m+\left(1+\delta \lambda^{\prime}\right)((\theta+\mu)-\mu m)\right\}}{m \mu+\left(1+\delta \lambda^{\prime}\right)((\theta+\mu)-\mu m)}\right] \\
& w_{3}=1
\end{aligned}
$$

Similarly, we can obtain the left eigenvector $\left(v_{1}, v_{2}, v_{3}\right)$ as $(0,0,1)$.

Using theorem 4.1 given in [11] the coefficients $a$ and $b$ can be computed as

$$
a=\sum_{k, i, j=1}^{3} v_{k} w_{i} w_{j} \frac{\partial^{2} f_{k}}{\partial x_{i} \partial x_{j}}\left(E_{0}, \lambda^{*}\right) \quad \text { and } \quad b=\sum_{k, i=1}^{3} v_{k} w_{i} \frac{\partial^{2} f_{k}}{\partial x_{i} \partial \beta}\left(E_{0}, \lambda^{*}\right)
$$

For the proposed system, the expression of a and b are obtained as

$$
\begin{aligned}
a= & v_{3} w_{3}\left[2 w_{1} \frac{\partial^{2} f_{3}}{\partial X_{1} \partial Y}\left(E_{0}, \lambda^{*}\right)+2 w_{2} \frac{\partial^{2} f_{3}}{\partial X_{2} \partial Y}\left(E_{0}, \lambda^{*}\right)\right] \\
= & -(\phi+\mu)\left[m \mu(1+\theta)+\left(1+\delta \lambda^{\prime}\right)(1+\theta)\{(\theta+\mu)-m \mu\}\right] \\
& -\left[m \mu^{2}+n \mu \theta-\delta \lambda^{\prime} n m \mu^{2}+\delta \lambda^{\prime} n \mu^{2}\right] \xi\left(\frac{\lambda^{*}}{\mu\left[m \mu+\left(1+\delta \lambda^{\prime}\right)\{(\theta+\mu)-\mu m\}\right]}\right)
\end{aligned}
$$

Similarly, we can obtain

$$
\begin{aligned}
b & =v_{3} w_{1} \frac{\partial^{2} f_{3}}{\partial X_{1} \partial \lambda}\left(E_{0}, \lambda^{*}\right)+v_{3} w_{2} \frac{\partial^{2} f_{3}}{\partial X_{2} \partial \lambda}\left(E_{0}, \lambda^{*}\right)+v_{3} w_{3} \frac{\partial^{2} f_{3}}{\partial Y \partial \lambda}\left(E_{0}, \lambda^{*}\right) \\
& =\frac{m A \mu+\left(1+\delta \lambda^{\prime}\right)\{(1-m) A(\theta+\mu)+\theta m A\}}{\mu(\theta+\mu)}
\end{aligned}
$$

From the expression of $a$ and $b$, it is evident that $a<0$ and $b>0$. This precludes the existence of backward bifurcation. Therefore, Eq. (7) does not possess a positive root for $R_{0}<1$.

\section{Stability Analysis}

This section comprises of stability results of two equilibrium points (disease free and endemic) of the proposed model system (1)-(3). We start with the stability analysis of disease free point and subsequently we study the stability of endemic equilibrium point.

\section{Disease Free Equilibrium Point}

First, we have performed a detailed local and global stability analysis of the disease free equilibrium.

\section{Local Stability}

The local stability of the disease free equilibrium point $E_{0}$ has been established by the following theorem.

Theorem 1 The disease free equilibrium point $E_{0}$ is locally asymptotically stable for $R_{0}<1$ and unstable for $R_{0}>1$ 
Proof As discussed in "Existence of Backward Bifurcation" section, jacobian matrix of the model system (1)-(3) around $E_{0}$ is

$$
J_{0}=\left[\begin{array}{ccc}
-(\theta+\mu) & 0 & -\beta X^{0}+n \xi \\
\theta & -\mu & -\lambda\left(1+\delta \lambda^{\prime}\right) P^{0}+(1-n) \xi \\
0 & 0 & \lambda X^{0}+\lambda\left(1+\delta \lambda^{\prime}\right) P^{0}-(\xi+\phi+\mu)
\end{array}\right]
$$

The characteristic polynomial of the matrix $J_{0}$ is

$$
\left(a_{11}-\Theta\right)\left(a_{22}-\Theta\right)\left(a_{33}-\Theta\right)=0
$$

where

$$
\begin{aligned}
& a_{11}=-(\theta+\mu) \\
& a_{22}=-\mu \\
& a_{33}=\lambda X^{0}+\lambda\left(1+\delta \lambda^{\prime}\right) P^{0}-(\xi+\phi+\mu)
\end{aligned}
$$

It is clear that all the three eigenvalues are negative iff $R_{0}<1$. Hence, the theorem.

\section{Global Stability}

To perform the global stability analysis of the disease free equilibrium we use the method developed by [10]. The model system (1) - (3) can be written as

$$
\begin{aligned}
& \frac{d X}{d t}=F(X, Z) \\
& \frac{d Z}{d t}=G(X, Z), G(X, 0)=0
\end{aligned}
$$

Here $X=\left(X_{1}, X_{2}\right) \in R^{2}$ represents (its components) the number of uninfected individuals and $Z=Z(Y) \in R$ denotes the number of infected individuals. According to this notation, the disease free equilibrium point is denoted by $Q_{0}=\left(X^{0}, 0\right)$.

Now as per the method given in [10], following two conditions will ensure global stability of the disease free equilibrium point

(H1) For $\frac{d X}{d t}=F(X, 0), X^{0}$ is globally asymptotically stable.

(H2) $G(X, Z)=B Z-\hat{G}(X, Z)$, where $\hat{G}(X, Z) \geq 0$ for $(X, Z) \in \Omega$

where $B=D_{Z} G\left(X^{0}, 0\right)$ is a M-matrix (matrix with non-negative off-diagonal elements) and $\Omega$ is the region defined in lemma 1 . Next, we state the following lemma.

Lemma 2 The fixed point $Q_{0}=\left(X^{0}, 0\right)$ is a globally asymptotically stable equilibrium of system (8)-(9), provided $R_{0}<1$ and assumptions (H1)-(H2) are satisfied.

Theorem 2 For $R_{0}<1$, the disease free equilibrium point is globally asymptotically stable.

Proof We begin by showing (H1) as

$$
F(X, 0)=\left[\begin{array}{c}
m A-(\theta+\mu) X \\
(1-m) A+\theta X-\mu P
\end{array}\right]
$$

The characteristic polynomial of the system is given by

$$
(\Theta+\mu)(\Theta+(\theta+\mu))=0
$$


There are two negative characteristic roots: $\Theta_{1}=-\mu$ and $\Theta_{2}=-(\theta+\mu)$. Hence $X=X^{0}$ is globally asymptotically stable.

Now we have

$$
\begin{aligned}
G(X, Z)= & {\left[\lambda X^{0}+\lambda\left(1+\delta \lambda^{\prime}\right) P^{0}-(\xi+\phi+\mu)\right] Y } \\
& -\left[\lambda Y\left(X^{0}-X\right)+\lambda\left(1+\delta \lambda^{\prime}\right)\left(P^{0}-P\right)\right]=B Z-\hat{G}(X, Z) .
\end{aligned}
$$

Here, $B$ is a M-matrix and $\hat{G}(X, Z) \geq 0$. Therefore, the conditions stated in $(\mathrm{H} 1)$ and $(\mathrm{H} 2)$ are satisfied. Hence the theorem.

\section{Endemic Equilibrium Point}

In this section, we will study the local and global stability of the endemic equilibrium point $E^{*}$ of the model system (1)-(3). First we will study the local stability of the endemic equilibrium point $E^{*}$ followed by the global stability.

\section{Local Stability}

An application of theorem 4.1 given in [11] to our model system and using the bifurcation coefficients a and b obtained in "Existence of Backward Bifurcation" section gives the conditions for local stability of endemic equilibrium point. The theorem can be stated as under

Theorem 3 The endemic equilibrium $E^{*}$ is locally asymptotically stable for $R_{0}>1$.

\section{Global Stability}

We will study the global stability of endemic equilibrium point $E^{*}$ by using the geometric approach $[36,37,48]$. Here, we briefly discuss the approach used in the present work. $D \subset R^{n}$ is an open and simply connected open set and $f \in C^{1}(D)$. Further, suppose that $\Psi(t)$ be a solution to the following system

$$
x^{\prime}=f(x)
$$

Let $P(x)$ be a $\left(\begin{array}{l}n \\ 2\end{array}\right) \times\left(\begin{array}{l}n \\ 2\end{array}\right)$ matrix valued function that is $C^{1}$ on $D$ and let that $B=P_{f} P^{-1}+$ $P J^{[2]} P^{-1}$. Here, the matrix $P_{f}$ is

$$
\left(p_{i j}(x)\right)_{f}=\left(\frac{\partial p_{i j}(x)}{\partial x}\right)^{T} \cdot f(x)=\nabla p_{i j} \cdot f(x)
$$

and $J^{[2]}$ is the second additive compound matrix obtained from the Jacobian matrix $J$.

Next, consider the following system

$$
\frac{d z}{d t}=Q(\Psi(t)) z
$$

Now, if Eq. (12) is stable then also the following second compound equation

$$
\frac{d \bar{z}}{d t}=J^{[2]}(\Psi(t)) \bar{z}
$$


as far as the $\Psi$ belongs to a set in which $\left|P^{-1}\right|$ is bounded. Further, a set $\bar{D}$ is absorbing with respect to Eq. (10) if solutions exist for all $t \geq 0$ and each bounded subset $D_{1}$ of $D$ satisfies $x\left(t, D_{1}\right) \subset \bar{D}$ for all $t$.

We also use the theorem 5 given in [39] to derive the global stability of the endemic equilibrium point.

The Jacobian matrix at an arbitrary point is

$$
M=\left[\begin{array}{ccc}
-\lambda Y-(\lambda+\mu) & 0 & -\lambda X+n \xi \\
\theta & -\lambda\left(1+\delta \lambda^{\prime}\right) Y-\mu & -\lambda\left(1+\delta \lambda^{\prime}\right) P+(1-n) \xi \\
\lambda Y & \lambda\left(1+\delta \lambda^{\prime}\right) Y & \lambda X+\lambda\left(1+\delta \lambda^{\prime}\right) P-(\xi+\phi+\mu)
\end{array}\right]
$$

The corresponding compound matrix is given as

$$
M^{[2]}=\left[\begin{array}{ccc}
M_{11} & -\lambda\left(1+\delta \lambda^{\prime}\right) X_{2}+(1-n) \xi \lambda X-n \xi \\
\lambda\left(1+\delta \lambda^{\prime}\right) Y & M_{22} & 0 \\
-\lambda Y & \theta & M_{33}
\end{array}\right]
$$

Here

$$
\begin{aligned}
& M_{11}=-\lambda Y-\lambda\left(1+\delta \lambda^{\prime}\right) Y-(\theta+2 \mu) \\
& M_{22}=-\lambda Y+\lambda X+\lambda\left(1+\delta \lambda^{\prime}\right) P-(\xi+\phi+\theta+2 \mu) \\
& M_{33}=-\lambda\left(1+\delta \lambda^{\prime}\right) Y+\lambda X+\lambda\left(1+\delta \lambda^{\prime}\right) P-(\xi+\phi+2 \mu)
\end{aligned}
$$

Let $P=\frac{1}{Y} I_{3}$. Where $I_{3}$ is the identity matrix of order 3. $P_{f} P^{-1}=-\frac{Y^{\prime}}{Y} I_{3}$, where $Y^{\prime}$ can be obtained from Eq. (3).

Next, $Q=P_{f} P^{-1}+P M^{[2]} P^{-1}$ is

$$
\left[\begin{array}{ccc}
Q_{11} & -\lambda\left(1+\delta \lambda^{\prime}\right) P+(1-n) \xi & \lambda X-n \xi \\
\lambda\left(1+\delta \lambda^{\prime}\right) Y & -\lambda Y-(\theta+\mu) & 0 \\
-\lambda Y & \theta & -\lambda\left(1+\delta \lambda^{\prime}\right) Y-\mu
\end{array}\right]
$$

Here, $Q_{11}=\left[-\lambda X-\lambda Y-\lambda\left(1+\delta \lambda^{\prime}\right) P-\lambda\left(1+\delta \lambda^{\prime}\right) Y-(\theta+\mu-\xi-\phi)\right]$.

Now, for some $z=\left(z_{1}, z_{2}, z_{3}\right)$, we define the following norm [4]

$$
\|z\|= \begin{cases}\max \left\{\left|z_{1}\right|+\left|z_{3}\right|,\left|z_{2}\right|+\left|z_{3}\right|\right\} & \text { if } z_{2} z_{3} \geq 0 \\ \max \left\{\left|z_{1}\right|+\left|z_{3}\right|,\left|z_{2}\right|\right\} & \text { if } z_{2} z_{3} \leq 0\end{cases}
$$

Now, we explore the existence of some $\chi>0$ so that $D_{+}\|z\| \leq-\chi\|z\|$. Based on the octant and norm, we have eight possible cases.

We assume that $\delta \lambda^{\prime}<1$. This inequality will help us to obtain the estimates on $D_{+}\|z\|$.

Case 1 If $0<z_{1} z_{2} z_{3}$ and $\left|z_{1}\right|+\left|z_{3}\right|>\left|z_{2}\right|+\left|z_{3}\right|$ then $\|z\|=\left|z_{1}\right|+\left|z_{3}\right|$ and

$$
\begin{aligned}
D_{+}\|z\|= & D_{+}\left(\left|z_{1}\right|+\left|z_{3}\right|\right)=z_{1}^{\prime}+z_{3}^{\prime} \\
= & {\left[-\lambda X-\lambda Y-\lambda\left(1+\delta \lambda^{\prime}\right) P-\lambda\left(1+\delta \lambda^{\prime}\right) Y-(\theta+\mu-\xi-\phi)\right] z_{1} } \\
& +\left(-\lambda\left(1+\delta \lambda^{\prime}\right) P+(1-n) \xi\right) z_{2}+(\lambda X-n \xi) z_{3}+(-\lambda Y) z_{1}+\theta z_{2} \\
& +\left(-\lambda\left(1+\delta \lambda^{\prime}\right) Y-\mu\right) z_{3} \\
\leq & {\left[-\lambda X-\lambda Y-\lambda\left(1+\delta \lambda^{\prime}\right) P-\lambda\left(1+\delta \lambda^{\prime}\right) Y-(\theta+\mu-\xi-\phi)\right]\left|z_{1}\right| } \\
& +\left(-\lambda\left(1+\delta \lambda^{\prime}\right) P+(1-n) \xi+\theta\right)\left|z_{2}\right|+\left(\lambda X-\lambda\left(1+\delta \lambda^{\prime}\right) Y-n \xi-\mu\right)\left|z_{3}\right|
\end{aligned}
$$


Since $z_{1}>z_{2}$, we get

$$
\begin{aligned}
D_{+}\|z\| \leq & {\left[-\lambda X-\lambda Y-\lambda\left(1+\delta \lambda^{\prime}\right) P-\lambda\left(1+\delta \lambda^{\prime}\right) Y-(\mu-\phi-2 \xi)\right]\left|z_{1}\right| } \\
& +\left(\lambda X-\lambda\left(1+\delta \lambda^{\prime}\right) Y-n \xi-\mu\right)\left|z_{3}\right| \\
D_{+}\|z\| \leq & \max \left[-\lambda X-\lambda Y-\lambda\left(1+\delta \lambda^{\prime}\right) Y-\lambda\left(1+\delta \lambda^{\prime}\right) P-(\mu-2 \xi-\phi),\right. \\
& \left.\lambda X-\lambda\left(1+\delta \lambda^{\prime}\right) Y-\mu-n \xi\right]\|z\|
\end{aligned}
$$

Case $20<z_{1} z_{2} z_{3}$ and $\left|z_{1}\right|+\left|z_{3}\right|<\left|z_{2}\right|+\left|z_{3}\right|$ then $\|z\|=\left|z_{2}\right|+\left|z_{3}\right|$ and

$$
\begin{aligned}
D_{+}\|z\|= & z_{2}^{\prime}+z_{3}^{\prime}=\left(\lambda\left(1+\delta \lambda^{\prime}\right) Y\right) z_{1}+(-\lambda Y-(\theta+\mu)) z_{2}+(-\lambda Y) z_{1} \theta z_{2} \\
& +\left(-\lambda\left(1+\delta \lambda^{\prime}\right) Y-\mu\right) z_{3}
\end{aligned}
$$

Now we have $z_{1}<z_{2}$, this becomes

$$
D_{+}\|z\| \leq\left[\lambda\left(1+\delta \lambda^{\prime}\right) Y-\lambda Y-\mu\right]\|z\|
$$

Case 3 If $z_{1}<0<z_{2}, z_{3}$ and $\left|z_{1}\right|+\left|z_{3}\right|>\left|z_{2}\right|+\left|z_{3}\right|$, then $\|z\|=\left|z_{1}\right|+\left|z_{3}\right|$ and

$$
\begin{aligned}
D_{+}\|z\|=-z_{1}^{\prime}+z_{3}^{\prime}= & \left(\lambda X+\lambda Y+\lambda\left(1+\delta \lambda^{\prime}\right) P+\lambda\left(1+\delta \lambda^{\prime}\right) Y+(\theta+\mu-\xi-\phi)\right) z_{1} \\
& +\left(\lambda\left(1+\delta \lambda^{\prime}\right) P-(1-n) \xi\right) z_{2}-(\lambda X-n \xi) z_{3} \\
& +(-\lambda Y) z_{1}+\theta z_{2}+\left(-\lambda\left(1+\delta \lambda^{\prime}\right) Y-\mu\right) z_{3} \\
= & \left(\lambda X+\lambda\left(1+\delta \lambda^{\prime}\right) P+\lambda\left(1+\delta \lambda^{\prime}\right) Y+(\theta+\mu-\xi-\phi)\right) z_{1} \\
& +\left(\lambda\left(1+\delta \lambda^{\prime}\right) P-(1-n) \xi+\theta\right) z_{2} \\
& -\left(\lambda X+\lambda\left(1+\delta \lambda^{\prime}\right) Y+\mu-n \xi\right) z_{3} \\
\leq & -\left(\lambda X+\lambda\left(1+\delta \lambda^{\prime}\right) P+\lambda\left(1+\delta \lambda^{\prime}\right) Y+(\theta+\mu-\xi-\phi)\right)\left|z_{1}\right| \\
& +\left(\lambda\left(1+\delta \lambda^{\prime}\right) P-(1-n) \xi+\theta\right)\left|z_{2}\right| \\
& -\left(\lambda X+\lambda\left(1+\delta \lambda^{\prime}\right) Y+\mu-n \xi\right)\left|z_{3}\right|
\end{aligned}
$$

Since $\left|z_{2}\right|<\left|z_{1}\right|$, we have

$$
\begin{aligned}
D_{+}\|z\| \leq & \max \left[-\lambda X-\lambda Y-\lambda\left(1+\delta \lambda^{\prime}\right) Y-(\mu-\xi-\phi),\right. \\
& \left.-\lambda X-\lambda\left(1+\delta \lambda^{\prime}\right) Y-(\mu-n \xi)\right]\|z\|
\end{aligned}
$$

Case 4 If $z_{1}<0<z_{2}, z_{3}$ and $\left|z_{1}\right|+\left|z_{3}\right|<\left|z_{2}\right|+\left|z_{3}\right|$, then $\|z\|=\left|z_{2}\right|+\left|z_{3}\right|$ and

$$
\begin{aligned}
D_{+}\|z\|= & z_{2}^{\prime}+z_{3}^{\prime}=\left(\lambda\left(1+\delta \lambda^{\prime}\right) Y\right) z_{1}+(-\lambda Y-(\theta+\mu)) z_{2}+(-\lambda Y) z_{1} \theta z_{2} \\
& +\left(-\lambda\left(1+\delta \lambda^{\prime}\right) Y-\mu\right) z_{3} \\
= & \left(\lambda\left(1+\delta \lambda^{\prime}\right) Y-\lambda Y\right) z_{1}+(-\lambda Y-\mu) z_{2}+\left(-\lambda\left(1+\delta \lambda^{\prime}\right) Y-\mu\right) z_{3} \\
\leq & \left(-\lambda\left(1+\delta \lambda^{\prime}\right) Y+\lambda Y\right)\left|z_{1}\right|+(-\lambda Y-\mu)\left|z_{2}\right|+\left(-\lambda\left(1+\delta \lambda^{\prime}\right) Y-\mu\right)\left|z_{3}\right|
\end{aligned}
$$

Since $\left|z_{1}\right|<\left|z_{2}\right|$, we get

$$
D_{+}\|z\| \leq-\mu\|z\|
$$


Case 5 If $z_{2}<0<z_{1}, z_{3}$ and $\left|z_{1}\right|+\left|z_{3}\right|>\left|z_{2}\right|$, then $\|z\|=\left|z_{1}\right|+\left|z_{3}\right|$ and

$$
\begin{aligned}
D_{+}\|z\|= & z_{1}^{\prime}+z_{3}^{\prime}=\left[-\lambda X-\lambda Y-\lambda\left(1+\delta \lambda^{\prime}\right) P-\lambda\left(1+\delta \lambda^{\prime}\right) Y-(\theta+\mu-\xi-\phi)\right] z_{1} \\
& +\left(-\lambda\left(1+\delta \lambda^{\prime}\right) P+(1-n) \xi\right) z_{2}+(\lambda X-n \xi) z_{3}+(-\lambda Y) z_{1} \\
& +\theta z_{2}+\left(-\lambda\left(1+\delta \lambda^{\prime}\right) Y-\mu\right) z_{3} \\
= & {\left[-\lambda X-\lambda Y-\lambda\left(1+\delta \lambda^{\prime}\right) P-\lambda\left(1+\delta \lambda^{\prime}\right) Y-(\theta+\mu-\xi-\phi)\right] z_{1} } \\
& +\left(-\lambda\left(1+\delta \lambda^{\prime}\right) P+(1-n) \xi+\theta\right) z_{2}+\left(\lambda X-\lambda\left(1+\delta \lambda^{\prime}\right) Y-\mu-n \xi\right) z_{3} \\
\leq & {\left[-\lambda X-\lambda Y-\lambda\left(1+\delta \lambda^{\prime}\right) P-\lambda\left(1+\delta \lambda^{\prime}\right) Y-(\theta+\mu-\xi-\phi)\right]\left|z_{1}\right| } \\
& -\left(-\lambda\left(1+\delta \lambda^{\prime}\right) P+(1-n) \xi+\theta\right)\left|z_{2}\right|+\left(\lambda X-\lambda\left(1+\delta \lambda^{\prime}\right) Y-\mu-n \xi\right)\left|z_{3}\right|
\end{aligned}
$$

Since $\left|z_{1}\right|+\left|z_{3}\right|>\left|z_{2}\right|$, we get

$$
\begin{gathered}
D_{+}\|z\| \leq \max \left[-\lambda X-\lambda Y-\lambda\left(1+\delta \lambda^{\prime}\right) Y-(\theta+\mu-\xi-\phi),\right. \\
\left.\lambda X+\lambda\left(1+\delta \lambda^{\prime}\right) P-\lambda\left(1+\delta \lambda^{\prime}\right) Y-\mu-n \xi\right]\|z\|
\end{gathered}
$$

Case 6 If $z_{2}<0<z_{1}, z_{3}$ and $\left|z_{1}\right|+\left|z_{3}\right|<\left|z_{2}\right|$, then $\|z\|=\left|z_{2}\right|$ and

$$
\begin{aligned}
& D_{+}\|z\|=-z_{2}^{\prime}=-\left(\lambda\left(1+\delta \lambda^{\prime}\right) Y\right) z_{1}-(-\lambda Y-(\theta+\mu)) z_{2} \\
& D_{+}\|z\| \leq-z_{2}^{\prime}=-\left(\lambda\left(1+\delta \lambda^{\prime}\right) Y\right)\left|z_{1}\right|+(-\lambda Y-(\theta+\mu))\left|z_{2}\right|
\end{aligned}
$$

Since $\left|z_{1}\right|+\left|z_{3}\right|<\left|z_{2}\right|$, we get

$$
D_{+}\|z\| \leq(-\lambda Y-(\theta+\mu))\left\|z_{2}\right\|
$$

Case 7 If $z_{3}<0<z_{1}, z_{2}$ and $\left|z_{1}\right|+\left|z_{3}\right|>\left|z_{2}\right|$, then $\|z\|=\left|z_{1}\right|+\left|z_{3}\right|$ and

$$
\begin{aligned}
D_{+}\|z\|= & z_{1}^{\prime}-z_{3}^{\prime}=\left[-\lambda X-\lambda Y-\lambda\left(1+\delta \lambda^{\prime}\right) P-\lambda\left(1+\delta \lambda^{\prime}\right) Y-(\theta+\mu-\xi-\phi)\right] z_{1} \\
& +\left(-\lambda\left(1+\delta \lambda^{\prime}\right) P+(1-n) \xi\right) z_{2}+(\lambda X-n \xi) z_{3}+(\lambda Y) z_{1} \\
& -\theta z_{2}+\left(\lambda\left(1+\delta \lambda^{\prime}\right) Y+\mu\right) z_{3} \\
D_{+}\|z\|= & {\left[-\lambda X-\lambda\left(1+\delta \lambda^{\prime}\right) P-\lambda\left(1+\delta \lambda^{\prime}\right) Y-(\theta+\mu-\xi-\phi)\right] z_{1} } \\
& +\left(-\lambda\left(1+\delta \lambda^{\prime}\right) P+(1-n) \xi-\theta\right) z_{2}+\left(\lambda X+\lambda\left(1+\delta \lambda^{\prime}\right) Y+\mu-n \xi\right) z_{3} \\
D_{+}\|z\| \leq & {\left[-\lambda X-\lambda\left(1+\delta \lambda^{\prime}\right) P-\lambda\left(1+\delta \lambda^{\prime}\right) Y-(\theta+\mu-\xi-\phi)\right]\left|z_{1}\right| } \\
& +\left(-\lambda\left(1+\delta \lambda^{\prime}\right) P+(1-n) \xi-\theta\right)\left|z_{2}\right|-\left(\lambda X+\lambda\left(1+\delta \lambda^{\prime}\right) Y+\mu-n \xi\right)\left|z_{3}\right|
\end{aligned}
$$

Since $\left|z_{1}\right|+\left|z_{3}\right|>\left|z_{2}\right|$, we get

$$
\begin{aligned}
D_{+}\|z\| \leq & \max \left[-\lambda X-\lambda\left(1+\delta \lambda^{\prime}\right) P-\lambda\left(1+\delta \lambda^{\prime}\right) Y-(\theta+\mu-2 \xi-\phi),\right. \\
& \left.\left.-\lambda X-\lambda\left(1+\delta \lambda^{\prime}\right) Y-\mu+\xi\right)\right]\|z\|
\end{aligned}
$$

Case 8 If $z_{3}<0<z_{1}, z_{2}$ and $\left|z_{1}\right|+\left|z_{3}\right|<\left|z_{2}\right|$, then $\|z\|=\left|z_{2}\right|$ and

$$
\begin{aligned}
D_{+}\|z\| & =z_{2}^{\prime}=\left(\lambda\left(1+\delta \lambda^{\prime}\right) Y\right) z_{1}+(-\lambda Y-(\theta+\mu)) z_{2} \\
& \leq\left(\lambda\left(1+\delta \lambda^{\prime}\right) Y\right)\left|z_{1}\right|+(-\lambda Y-(\theta+\mu))\left|z_{2}\right|
\end{aligned}
$$

Since $\left|z_{1}\right|+\left|z_{3}\right|<\left|z_{2}\right|$, we get

$$
D_{+}\|z\| \leq\left(\lambda\left(1+\delta \lambda^{\prime}\right) Y-\lambda Y-(\theta+\mu)\right)\|z\|
$$


Combining the results of the four different cases presented above and the remaining four cases, we state the following theorem

Theorem 4 For $R_{0}>1$, the endemic equilibrium point $E^{*}$ is globally asymptotically stable provided the following inequality holds

$$
\max \left(\chi_{1}, \chi_{2}, \chi_{3}, \chi_{4}\right)<-\chi
$$

where

$$
\begin{aligned}
\chi_{1}= & \max \left[-\lambda X-\lambda Y-\lambda\left(1+\delta \lambda^{\prime}\right) Y-\lambda\left(1+\delta \lambda^{\prime}\right) P-(\mu-2 \xi-\phi),\right. \\
& \left.\lambda X-\lambda\left(1+\delta \lambda^{\prime}\right) Y-\mu-n \xi\right] \\
\chi_{2}= & \max \left[-\lambda X-\lambda Y-\lambda\left(1+\delta \lambda^{\prime}\right) Y-(\mu-\xi-\phi),\right. \\
& \left.-\lambda X-\lambda\left(1+\delta \lambda^{\prime}\right) Y-(\mu-n \xi)\right] \\
\chi_{3}= & \max \left[-\lambda X-\lambda Y-\lambda\left(1+\delta \lambda^{\prime}\right) Y-(\theta+\mu-\xi-\phi),\right. \\
& \left.\lambda X+\lambda\left(1+\delta \lambda^{\prime}\right) P-\lambda\left(1+\delta \lambda^{\prime}\right) Y-\mu-n \xi\right] \\
\chi_{4}= & \max \left[-\lambda X-\lambda\left(1+\delta \lambda^{\prime}\right) P-\lambda\left(1+\delta \lambda^{\prime}\right) Y-(\theta+\mu-2 \xi-\phi),\right. \\
& \left.\left.-\lambda X-\lambda\left(1+\delta \lambda^{\prime}\right) Y-\mu+\xi\right)\right]
\end{aligned}
$$

and $\chi$ is a positive constant.

\section{Numerical Simulation}

We perform several numerical experiments to study the dynamics of the disease when population is influenced by environmental pollution. We also try to establish the feasibility of analytical findings using numerical simulation. Since, the field data is not available for study, therefore most of the parameter values are chosen from the literature $[21,33,40]$. The set of parameter values considered for simulation is

$$
\begin{aligned}
& A=200, m=0.8, \lambda=0.00002, \delta=0.3, \lambda^{\prime}=0.1, \theta=0.004, \\
& \mu=0.035, \xi=0.012, \phi=0.01, n=0.7
\end{aligned}
$$

For the above set of parameters, the value of basic reproduction number is 2.0220. Moreover, the eigenvalues of the Jacobian matrix corresponding to endemic equilibrium point are, $-0.0419+0.0205 i,-0.0419-0.0205 i$ and -0.0864 . Since all the eigenvalues have negative real parts, hence the system is locally asymptotically stable. To establish the nonlinear stability, we plot the trajectories of infected population $Y$ versus susceptible population $X$ in Fig. 2 and infected population $Y$ versus the stressed population $P$ in Fig. 3. It is easy to observe that all the trajectories converge to the endemic equilibrium point $E^{*}$. Therefore, the endemic equilibrium point is stable in $X-Y$ and $P-Y$ phase planes.

The primary aim of the present study is to investigate the impact of environmental pollution on the spread of disease. Therefore, we perform a comprehensive numerical study to gauge the impact of pollution related parameters on the disease dynamics. As explained earlier (in "Mathematical Model" section), there are two possible modes through which individuals can move into the stressed class $P$. These modes can be pointed out as under:

(i) A fraction of the newborns will directly enter into $P$ due to the parental exposure to toxic chemicals present in the environment. 


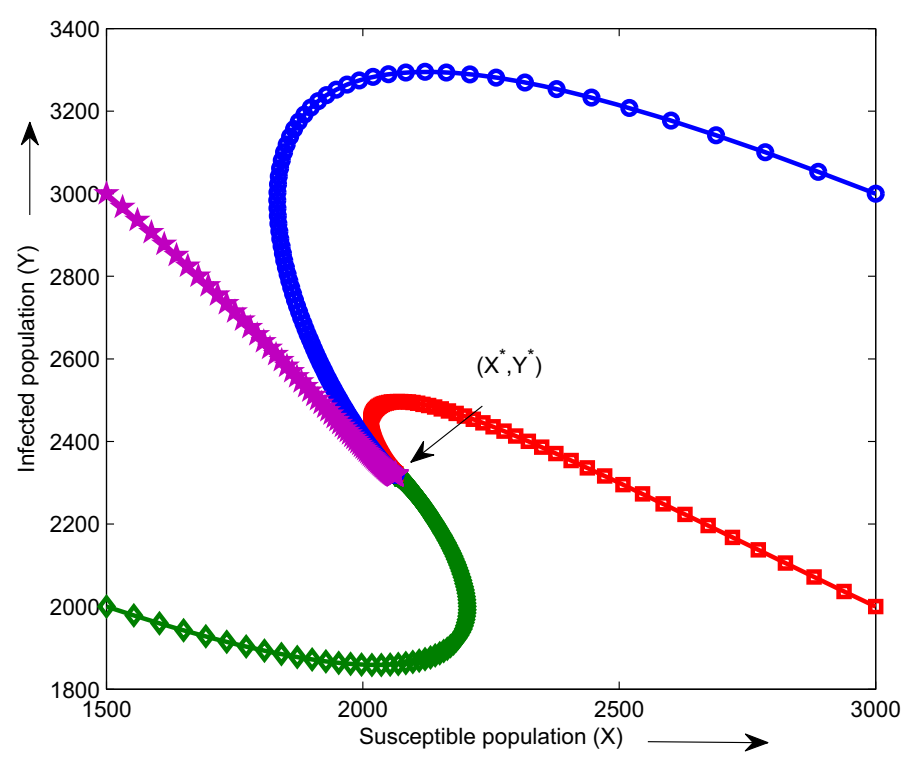

Fig. 2 Global stability of endemic equilibrium point $E^{*}$ in $X-Y$ plane

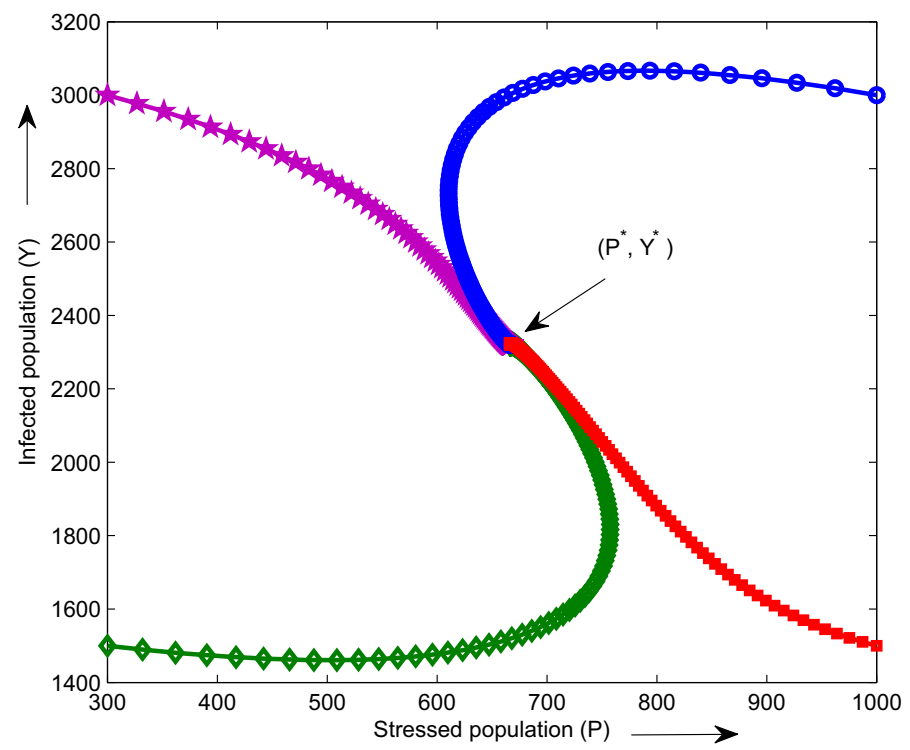

Fig. 3 Global stability of endemic equilibrium point $E^{*}$ in $P-Y$ plane

(ii) A part of susceptible individuals $(\theta X)$ will move to the stressed class due to their frequent exposure to pollutants.

Therefore, we perform numerical simulations which involve variation in $R_{0}$ and other pollution related parameters. In Fig. 4, the effect of variations in $\theta$ and $\lambda^{\prime}$ have been studied. From the figure, we observe that for smaller magnitude of $\theta$, the effect of variation of $\lambda^{\prime}$ is small. However, as the value of $\theta$ increases, the value of $R_{0}$ also increases significantly. The 


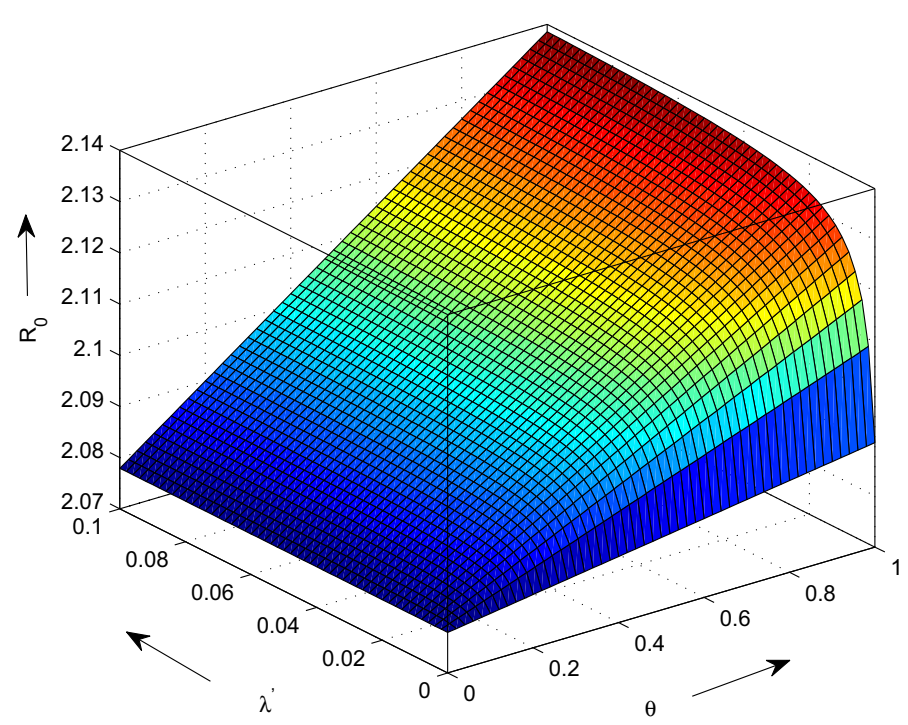

Fig. 4 Surface plot showing simultaneous effect of $\theta$ and $\lambda^{\prime}$ on $R_{0}$

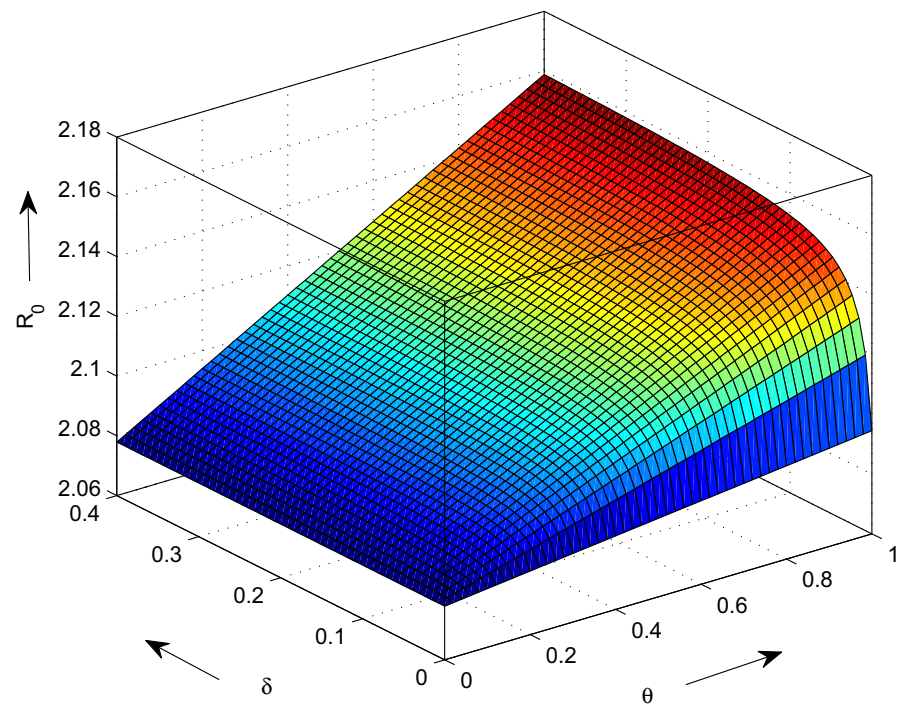

Fig. 5 Surface plot showing simultaneous effect of $\theta$ and $\delta$ on $R_{0}$

variation of $R_{0}$ with change in values of $\theta$ and $\delta$ has been studied in Fig. 5. The figure clearly reflects that the value of $R_{0}$ increases with an increase in the value of $\theta$ and $\delta$, which are the parameters showing effects of environmental pollution, increases. In Fig. 6, we have studied the impact of $m$ and $\lambda^{\prime}$ on $R_{0}$. The figure clearly reflects that the value of $R_{0}$ increases rapidly with an increase in $\lambda^{\prime}$ and decrease in $m$. On the same line, we have studied the impact of variation of $\delta$ and $m$ on $R_{0}$ in Fig. 7. The rise in the value of $R_{0}$ is easy to observe from this figure. Figures 4, 5, 6 and 7 give a clear idea that environmental pollution has consequential influence on the dynamics of the disease. 


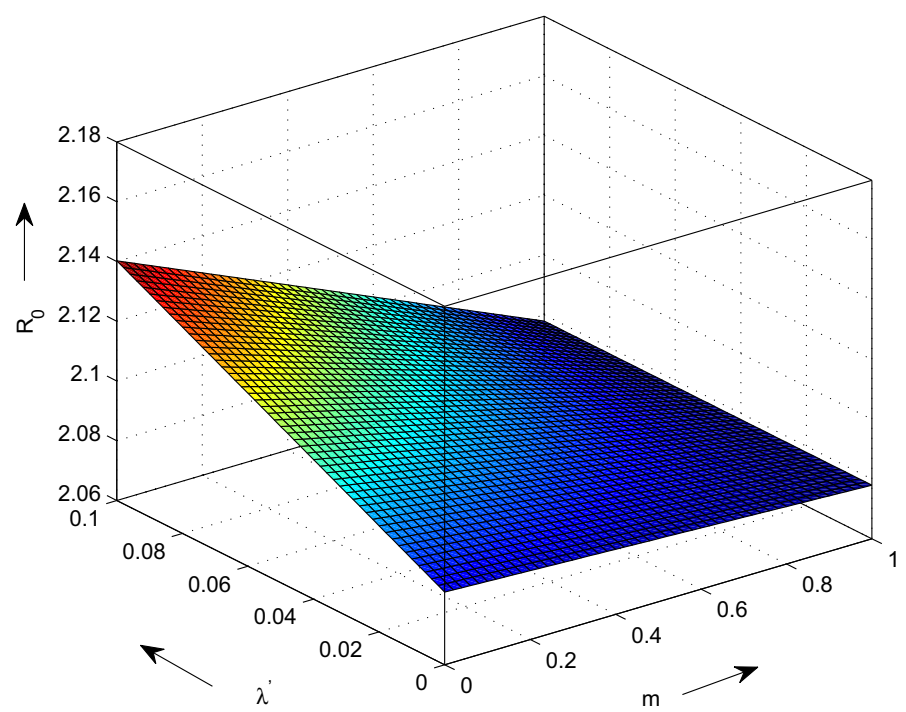

Fig. 6 Surface plot showing simultaneous effect of $m$ and $\lambda^{\prime}$ on $R_{0}$

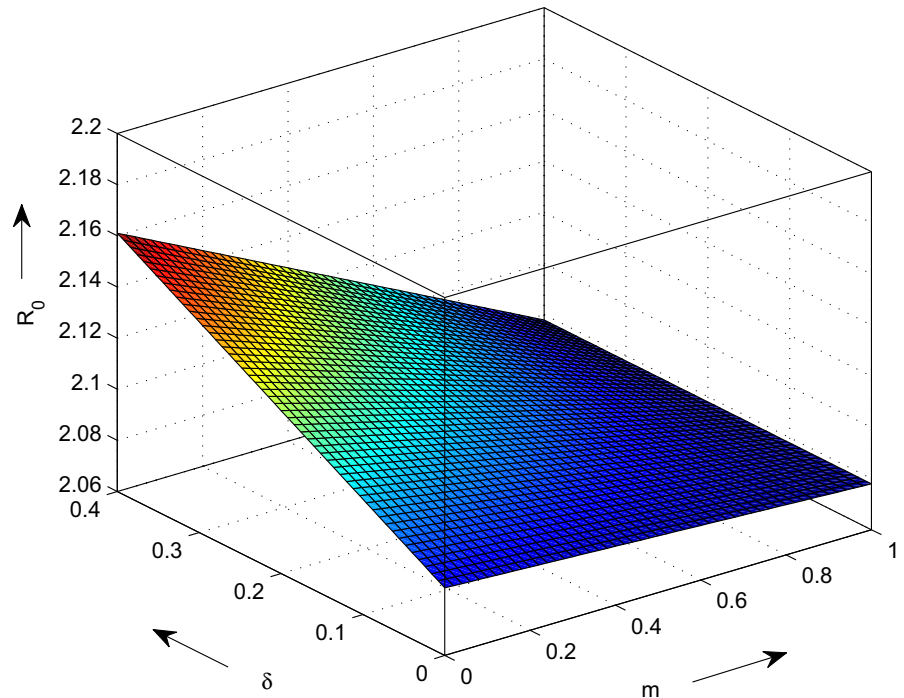

Fig. 7 Surface plot showing simultaneous effect of $m$ and $\delta$ on $R_{0}$

To get a clear view and to support our claim, fluctuations in the infected population $(Y)$ with respect to $\delta$ and $\lambda^{\prime}$, for different values of $m$ and $\theta$ have been studied. Figure 8 demonstrates the effect of $\delta$ on $Y$. It is easy to observe from this figure, that number of infected individuals increases with an increase in the value of $\delta$ and decrease in the value of $m$. Similar behavior has been observed for $\lambda^{\prime}$ (see Fig. 9). Figure 10 shows fluctuations in infected population with variations in $\delta$, for different values of $\theta$. We conclude from the figure that the number of infected individuals rises with an increase in $\delta$ and $\theta$. Same behavior of infected individuals has been observed for $\lambda^{\prime}$ and $\theta$ (see Fig. 11). 


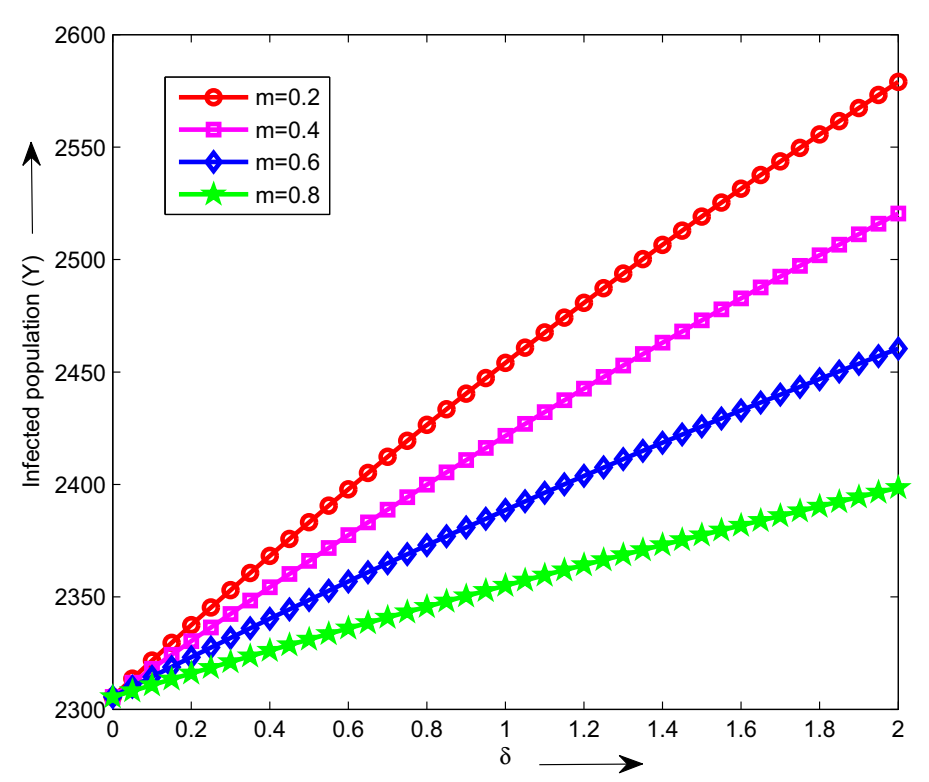

Fig. 8 Variation of infected population with $\delta$ for different values of $m$

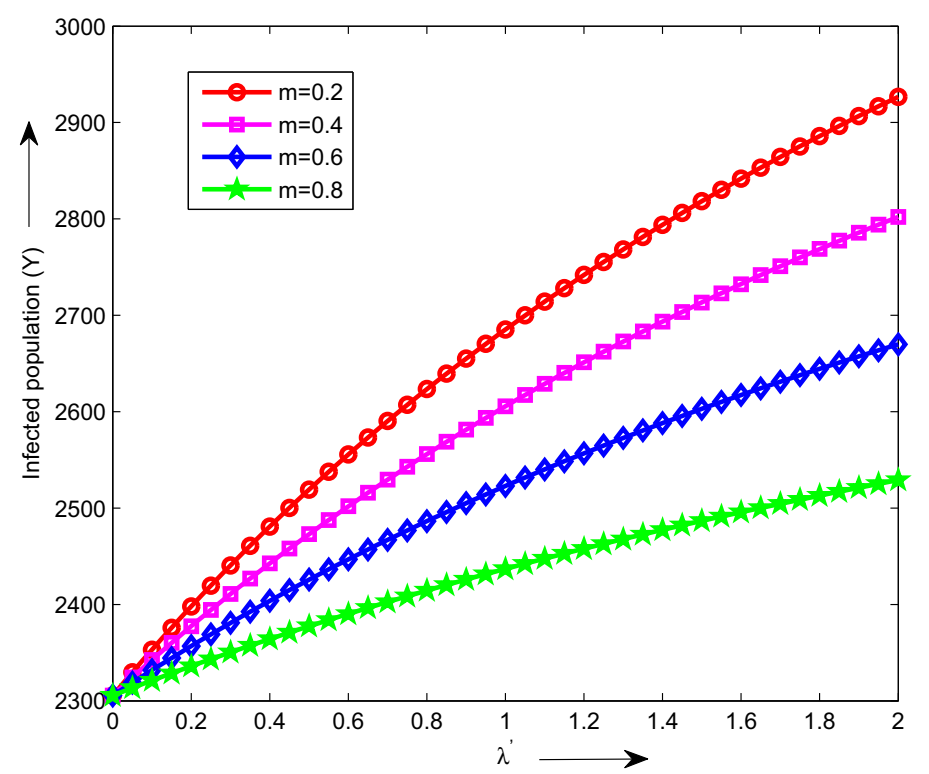

Fig. 9 Variation of infected population with $\lambda^{\prime}$ for different values of $m$

The numerical simulation also brought forth an interesting fact that the influence of pollution on disease is much more severe when the newborns contribute more in the stressed populations as compared to grown up susceptibles. A small increase in the transmission rate of susceptibles into the stressed compartment, leads to an increase in the infected population as seen in Figs. 9 and 10. Whereas, a small increase in the fraction of newborns who are 


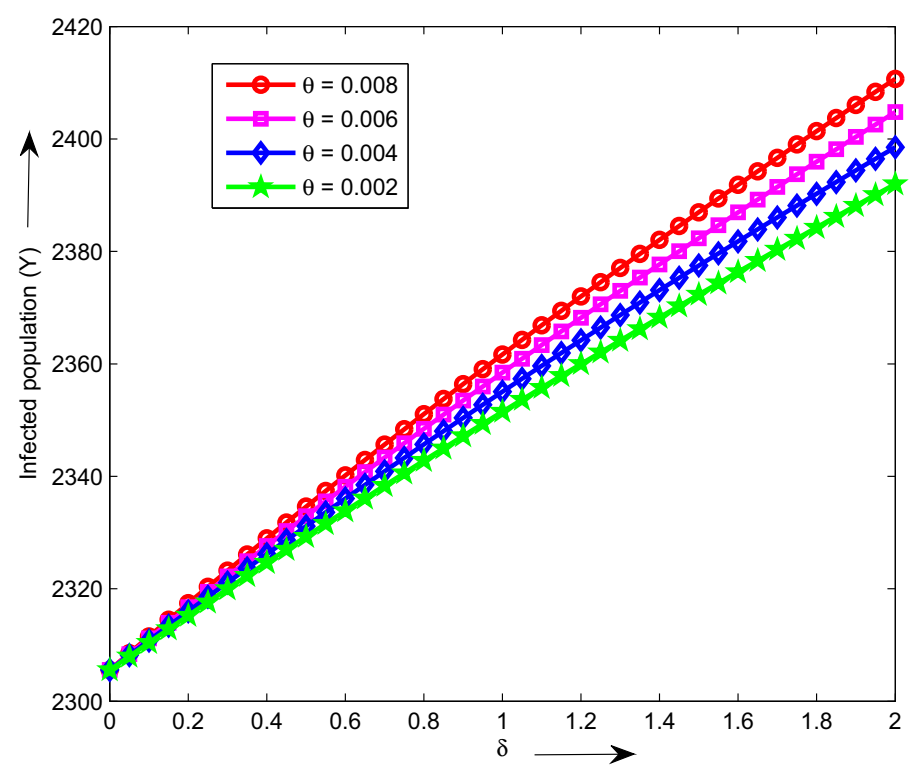

Fig. 10 Variation of infected population with $\delta$ for different values of $\theta$

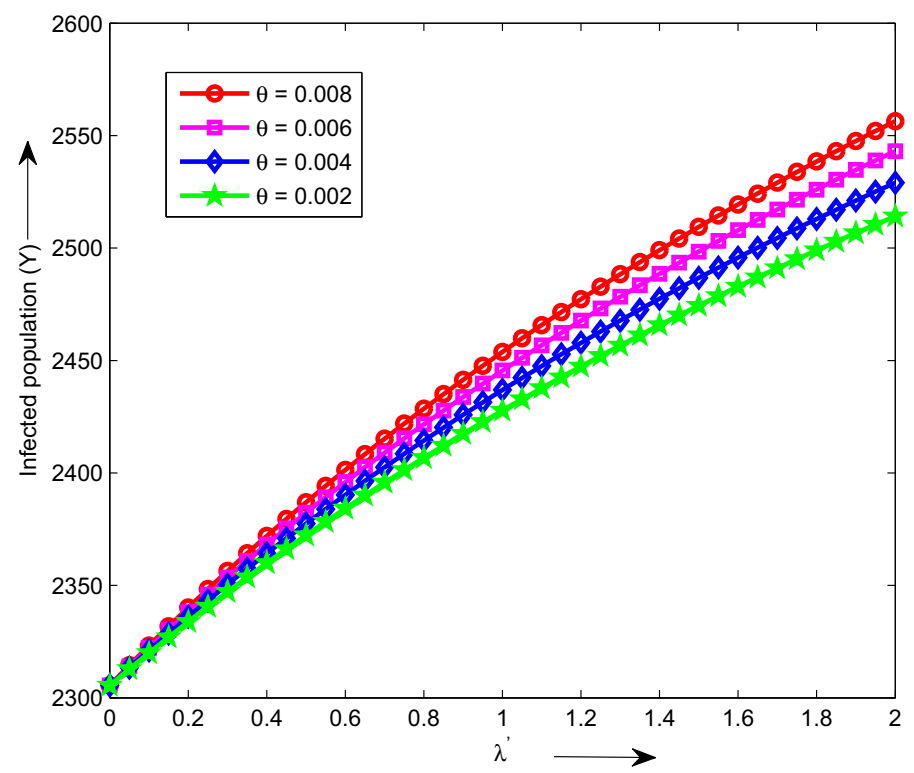

Fig. 11 Variation of infected population with $\lambda^{\prime}$ for different values of $\theta$

exposed to pollution causes a significant increase in the infected population which is relatively more than the increase due to $\theta$, see Figs. 8, 9, 10 and 11 . The variation of infected population in the presence and absence of environmental stress has been investigated in Fig. 12. From this figure, it is evident that the number of infected individuals remains higher in the presence of stress during the course of disease. 


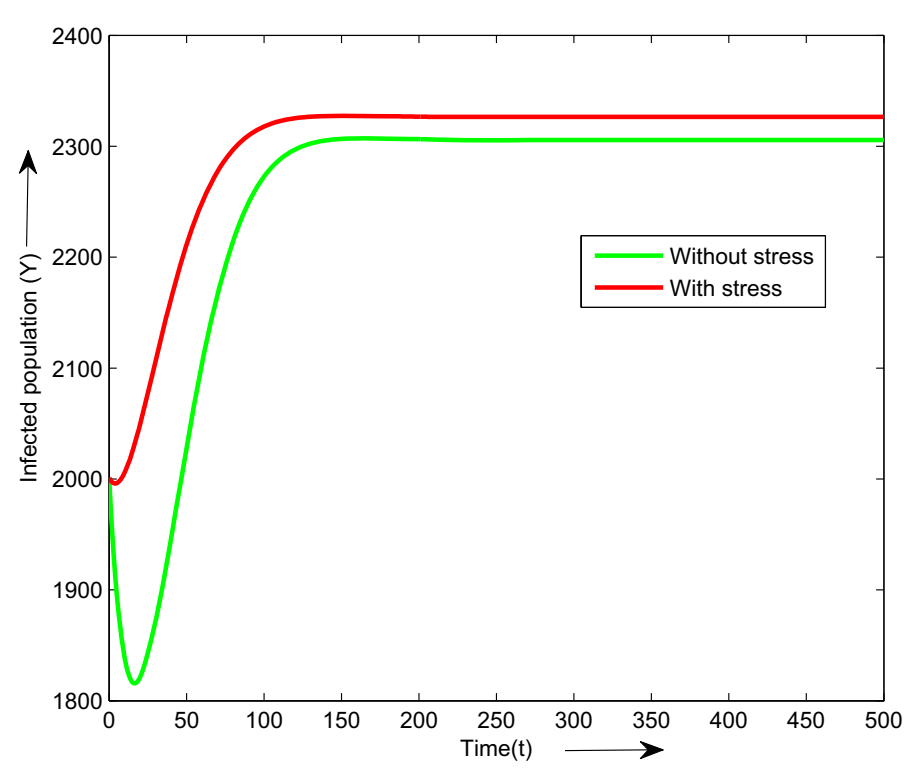

Fig. 12 Variation of infected population with time in the presence and absence of stress

This observation is very useful and a new finding which suggests that consequences of exposure of newborns to the pollution are relatively more severe. Therefore measures must be taken to protect newborns from being exposed to the pollution.

\section{Sensitivity Analysis}

Sensitivity analysis enables us to determine the relative change in a state variable when a parameter changes [13]. The normalized forward sensitivity index is defined as the ratio of the relative change in the variable to the corresponding relative change in the parameter. When the variable is a differentiable function of the parameter, then partial derivatives are also helpful to calculate the sensitivity index.

As the basic reproduction number $R_{0}$ enables us to determine the dynamics of the model, we perform the sensitivity analysis to study how sensitive $R_{0}$ is with the uncertainties in the estimation of the parameters involved in the model.

The following expression for the sensitivity is used in our study

$$
\psi_{s}^{R_{0}}=\frac{s}{R_{0}} \times \frac{\partial R_{0}}{\partial s}
$$

A negative sensitivity index indicates that the parameter and $R_{0}$ are inversely proportional. Similarly, a positive sensitivity index denotes that the value of $R_{0}$ increases with increase in the value of parameter.

The calculated sensitivity indices for $R_{0}$ (using the parameter values given in "Numerical Simulation" section) are presented in Table 2.

From Table 2, we observe that an increase or decrease in the value of $\lambda$ or $A$ will result in increase or decrease in the same proportion in $R_{0}$ as they are directly proportional. We have found that $\psi_{\theta}^{R_{0}}, \psi_{\delta}^{R_{0}}, \psi_{\lambda^{\prime}}^{R_{0}}$ are all positive quantities hence an increase in $\theta, \delta$ and $\lambda^{\prime}$ 
Table 2 Sensitivity analysis of $R_{0}$

\begin{tabular}{|c|c|c|}
\hline Parameter & Expression of the sensitivity index & Value of the sensitivity index \\
\hline A & 1 & 1 \\
\hline$\lambda$ & 1 & 1 \\
\hline$\theta$ & $\theta\left(\frac{\delta \lambda^{\prime} m \mu}{(\theta+\mu)\left[m+\left(1+\delta \lambda^{\prime}\right)((\theta+\mu)-m \mu)\right]}\right)$ & 0.0001 \\
\hline$m$ & $-m\left(\frac{\delta \lambda^{\prime} \mu}{m+\left(1+\delta \lambda^{\prime}\right)[(\theta+\mu)-\mu m]}\right)$ & -0.0010 \\
\hline$\delta$ & $\delta\left(\frac{\lambda^{\prime}(\theta+\mu-\mu m)}{m \mu+\left(1+\delta \lambda^{\prime}\right)[(\theta+\mu)-\mu m]}\right)$ & 0.0084 \\
\hline$\lambda^{\prime}$ & $\lambda^{\prime}\left(\frac{\delta(\theta+\mu-\mu m)}{m \mu+\left(1+\delta \lambda^{\prime}\right)[(\theta+\mu)-\mu m]}\right)$ & 0.0087 \\
\hline$\mu$ & a & -0.7139 \\
\hline$\xi$ & $-\frac{\xi}{(\xi+\phi+\mu)}$ & -0.1818 \\
\hline$\phi$ & $-\frac{\phi}{(\xi+\phi+\mu)}$ & -0.1818 \\
\hline \multicolumn{3}{|c|}{$\mu(\theta+\mu)(\xi+\phi+\mu)\left[\lambda m A+\lambda\left(1+\delta \lambda^{\prime}\right) A-\lambda\left(1+\delta \lambda^{\prime}\right) m\right]$} \\
\hline
\end{tabular}

will cause a corresponding direct increase in $R_{0}$. However, the magnitude of $\psi_{\theta}^{R_{0}}<<1$, hence the parameter $\theta$ is relatively less sensitive than $\delta$ and $\lambda^{\prime}$. It is also evident from the Table 2 that $\psi_{m}^{R_{0}}, \psi_{\mu}^{R_{0}}, \psi_{\xi}^{R_{0}}$ and $\psi_{\phi}^{R_{0}}$ are negative valued quantities. Hence $R_{0}$ decreases as the values of $m, \mu, \xi$ and $\phi$ increases and vice versa.

Our sensitivity analysis also indicates that $R_{0}$ is more sensitive to $m$ as compared to $\theta$. Hence, we can conclude that increase in the proportion of newborns in the stressed compartment makes the scenario worst.

\section{Conclusion}

In this paper, we propose a SIS type epidemiological model to understand the role of environmental pollution on the spread of infectious diseases. The Susceptible population is divided into two categories, namely susceptible and stressed. The analysis, both analytical as well as numerical, performed in this study, brought forth the fact that pollution has significant impact on the spread of disease. The expression of the basic reproduction number $R_{0}$, defined as a threshold quantity which governs the dynamics of an epidemic model, involves parameters representing environmental pollution (e.g $\delta$ and $\lambda^{\prime}$ ). Therefore, expression of $R_{0}$, itself, gives a hint regarding the role of environmental pollution in the spread of an infectious disease. The variation of $R_{0}$ with respect to the parameters $\delta, \lambda^{\prime}, \theta$ and $m$ (see Figs. 4, 5, 6, 7) shows a rise in the value of $R_{0}$ as value of these parameters increase. However, a rise in the value of $R_{0}$, with respect to the parameters of stress, is very sharp when the value of $m$ decreases as compared to increase in $\theta$. Similarly, an increase in the number of infected individuals has been observed with an increase in the value of pollution parameters $\delta$ and $\lambda^{\prime}$ which represent environmental pollution. We conclude from our study that environmental pollution plays a significant role in the spread of infectious diseases. Although, the pollution affects all 
individuals but the situation get worse when newborns and infants come under its influence. Figures 8 and 9, demonstrate that rise in infected population is relatively high when there is a decrease in value of parameter $m$. Hence, we conclude that prenatal exposure to toxic substances results in an unhealthy life of newborns. This is a new finding which has not been studied till date in the literature. Similarly, different surface plots of $R_{0}$, show a steep rise in the value of $R_{0}$ with respect to $m, \delta$ and $\lambda^{\prime}$.

The epidemiological insights provided by the present study can be summarized as follows

(i) Environmental pollution not only causes harmful effects and non-infectious diseases (like asthma, some types of cancer etc.) but also play an active role in the dynamics of an infectious disease.

(ii) The presence of pollution related parameters in the expression of basic reproduction number itself gives the hint that pollution definitely affects the long term dynamics of the disease.

(iii) The size of the epidemic increases with the level of pollution and number density of stressed individuals.

(iv) An increase in the rate of induction of newborns in the stressed class causes more serious impact in the spread of disease as compared to the movement of grown up susceptibles to the stressed class.

(v) The present study is, to the best of our knowledge, the maiden mathematical efforts in this direction. Further, The results of this study are in line with the ecological study carried in [35].

Thus, the findings of our study clearly indicates that the spread of infectious diseases turns out to be more serious in the presence of environmental pollution. This is an important epidemiological finding of the present study and will certainly helps the government agencies and organizations.

\section{Challenges and Future Scope}

From the above discussion, we can conclude that the present study successfully demonstrates the impact of environmental pollution on the disease dynamics. But, the application of the study in real scenario is interdicted by various challenges. We will point out certain issues which need to be addressed before the application of the present study in real world. However, many ecological and biological studies are also sharing the same limitations. The highlighted points or issues not only help us in the course of the present study but also contribute in the development of the conducive environment and living conditions. First, we list the above mentioned issues followed by the work that were carried out in that direction

(i) The primary limitation is the measurement of the pollution $(\delta)$.

(ii) The key assumption of the present study is that the presence of pollution increases the susceptibility of the individuals. But, to measure the increase in the susceptibility is itself a challenge and needs some further biological/immunological exploration.

(iii) Moreover, an increase in the susceptibility varies for different diseases. The susceptibility depends on the type of diseases and type of environmental pollutants to which the population is exposed.

(iv) The pollution level varies drastically as we move from one region to the other. Therefore, the inclusion of geographical and ecological factors will make the study more realistic. 
(v) The accuracy of the data is the key to success of the present study. But, the collection of such data needs a huge expenditure which is an impediment in the implementation of current study.

The limitations and challenges of the present study provokes the need of a better system which can detect and monitor the type and level of pollutants present in the environment. The implementation of such robust system will certainly enhance our understanding.

As the level of pollution increases rapidly and the combined dynamics of pollution and infectious diseases are yet to be explored, the present study requires multidimensional investigations from wide group of researchers including environmentalists and medical scientists. The compartmental models, available in the literature, can be modified by adding an extra compartment of pollution affected individuals. The inclusion of such compartments will certainly enhance our knowledge and understanding of the disease. Therefore, the present study opens a new window of work for biologists, immunologists, eco-epidemiologists and mathematicians working in epidemic modeling.

Acknowledgements The current research of N. Kumari is supported by IIT, Mandi under the Project IITM/SG/NK/008.

\section{References}

1. Alexander, M.E., Moghadas, S.M.: Periodicity in an epidemic model with a generalized non-linear incidence. Math. Biosci. 189(1), 75-96 (2004)

2. Alexander, M.E., Moghadas, S.M.: Bifurcation analysis of an SIRS epidemic model with generalized incidence. SIAM J. Appl. Math. 65(5), 1794-1816 (2005)

3. Allen, L.J.S., Brauer, F., Van den Driessche, P., Wu, J.: Mathematical Epidemiology. Springer, Berlin (2008)

4. Arino, J., Connell McCluskey, C., van den Driessche, P.: Global results for an epidemic model with vaccination that exhibits backward bifurcation. SIAM J. Appl. Math. 64(1), 260-276 (2003)

5. Brauer, F.: Backward bifurcations in simple vaccination models. J. Math. Anal. Appl. 298(2), 418-431 (2004)

6. Brisbois, B.W., Ali, S.H.: Climate change, vector-borne disease and interdisciplinary research: social science perspectives on an environment and health controversy. EcoHealth 7(4), 425-438 (2010)

7. Cai, L.-M., Li, X.-Z.: Analysis of a SEIV epidemic model with a nonlinear incidence rate. Appl. Math. Model. 33(7), 2919-2926 (2009)

8. Cai, L.-M., Li, X.-Z.: Global analysis of a vector-host epidemic model with nonlinear incidences. Appl. Math. Comput. 217(7), 3531-3541 (2010)

9. Capasso, V., Capasso, V.: Mathematical Structures of Epidemic Systems, vol. 88. Springer, Berlin (1993)

10. Castillo-Chávez, C., Blower, S., Driessche, P., Kirschner, D., Yakubu, A.-A.: Mathematical Approaches for Emerging and Reemerging Infectious Diseases: Models, Methods, and Theory, vol. 126. Springer, Berlin (2002)

11. Castillo-Chavez, C., Song, B.: Dynamical models of tuberculosis and their applications. Math. Biosci. Eng. 1(2), 361-404 (2004)

12. Chitnis, N., Cushing, J.M., Hyman, J.M.: Bifurcation analysis of a mathematical model for malaria transmission. SIAM J. Appl. Math. 67(1), 24-45 (2006)

13. Chitnis, N., Hyman, J.M., Cushing, J.M.: Determining important parameters in the spread of malaria through the sensitivity analysis of a mathematical model. Bull. Math. Biol. 70(5), 1272-1296 (2008)

14. Codeço, C.T.: Endemic and epidemic dynamics of cholera: the role of the aquatic reservoir. BMC Infect. Dis. 1(1), 1 (2001)

15. Cui, J., Sun, Y., Zhu, H.: The impact of media on the control of infectious diseases. J. Dyn. Differ. Equ. 20(1), 31-53 (2008)

16. Dobson, A.: Climate variability, global change, immunity, and the dynamics of infectious diseases. Ecology 90(4), 920-927 (2009)

17. Dubey, B.: A model for the effect of pollutant on human population dependent on a resource with environmental and health policy. J. Biol. Syst. 18(03), 571-592 (2010) 
18. Eckalbar, J.C., Eckalbar, W.L.: Dynamics of an epidemic model with quadratic treatment. Nonlinear Anal. Real World Appl. 12(1), 320-332 (2011)

19. Esteva, L., Vargas, C.: Analysis of a dengue disease transmission model. Math. Biosci. 150(2), 131-151 (1998)

20. Freedman, H.I., So, J.W.-H.: Global stability and persistence of simple food chains. Math. Biosci. 76(1), 69-86 (1985)

21. Ghosh, M., Chandra, P., Sinha, P., Shukla, J.B.: Modelling the spread of bacterial infectious disease with environmental effect in a logistically growing human population. Nonlinear Anal. Real World Appl. 7(3), 341-363 (2006)

22. Grandjean, P., Bellinger, D., Bergman, Å., Cordier, S., Davey-Smith, G., Eskenazi, B., Gee, D., Gray, K., Hanson, M., Van Den Hazel, P., et al.: The faroes statement: human health effects of developmental exposure to chemicals in our environment. Basic Clin. Pharmacol. Toxicol. 102(2), 73-75 (2008)

23. Greenhalgh, D., Rana, S., Samanta, S., Sardar, T., Bhattacharya, S., Chattopadhyay, J.: Awareness programs control infectious disease-multiple delay induced mathematical model. Appl. Math. Comput. 251, 539-563 (2015)

24. Hallam, T.G., Clark, C.E., Lassiter, R.R.: Effects of toxicants on populations: a qualitative approach I. Equilibrium environmental exposure. Ecol. Model. 18(3), 291-304 (1983)

25. Hallam, T.G., De Luna, J.T.: Effects of toxicants on populations: a qualitative: approach III. Environmental and food chain pathways. J. Theor. Biol. 109(3), 411-429 (1984)

26. Heesterbeek, J.A.P.: Mathematical Epidemiology of Infectious Diseases: Model Building, Analysis and Interpretation, vol. 5. Wiley, Hoboken (2000)

27. Hertz-Picciotto, I., Park, H.-Y., Dostal, M., Kocan, A., Trnovec, T., Sram, R.: Prenatal exposures to persistent and non-persistent organic compounds and effects on immune system development. Basic Clin. Pharmacol. Toxicol. 102(2), 146-154 (2008)

28. Hoshen, M.B., Morse, A.P.: A weather-driven model of malaria transmission. Malaria J. 3(1), 1 (2004)

29. Huaping, L., Zhien, M.: The threshold of survival for system of two species in a polluted environment. J. Math. Biol. 30(1), 49-61 (1991)

30. Kermack, W.O., McKendrick, A.G.: A contribution to the mathematical theory of epidemics. In: Proceedings of the Royal Society of London A: Mathematical, Physical and Engineering Sciences, vol. 115, pp. 700-721. The Royal Society (1927)

31. Khan, R.A.: Parasitism in marine fish after chronic exposure to petroleum hydrocarbons in the laboratory and to the exxon valdez oil spill. Bull. Environ. Contam. Toxicol. 44(5), 759-763 (1990)

32. Kribs-Zaleta, C.M., Velasco-Hernandez, J.X.: A simple vaccination model with multiple endemic states. Math. Biosci. 164(2), 183-201 (2000)

33. Kumari, N., Sharma, S.: Does water disinfectant play a supportive role in the spread of infectious disease? A mathematical study. Nat. Resour. Model. 29(2), 259-288 (2016)

34. Lafferty, K.D.: The ecology of climate change and infectious diseases. Ecology 90(4), 888-900 (2009)

35. Lafferty, K.D., Holt, R.D.: How should environmental stress affect the population dynamics of disease? Ecol. Lett. 6(7), 654-664 (2003)

36. Li, M.Y., Muldowney, J.S.: A geometric approach to global-stability problems. SIAM J. Math. Anal. 27(4), 1070-1083 (1996)

37. Li, Y., Muldowney, J.S.: On Bendixson's criterion. J. Differ. Equ. 106(1), 27-39 (1993)

38. McCluskey, C.C.: Complete global stability for an sir epidemic model with delay-distributed or discrete. Nonlinear Anal. Real World Appl. 11(1), 55-59 (2010)

39. McCluskey, C.C., van den Driessche, P.: Global analysis of two tuberculosis models. J. Dyn. Differ. Equ. 16(1), 139-166 (2004)

40. Misra, A.K., Sharma, A., Shukla, J.B.: Modeling and analysis of effects of awareness programs by media on the spread of infectious diseases. Math. Comput. Model. 53(5), 1221-1228 (2011)

41. Nakata, Y., Kuniya, T.: Global dynamics of a class of seirs epidemic models in a periodic environment. J. Math. Anal. Appl. 363(1), 230-237 (2010)

42. Patz, J.A., Githeko, A.K., McCarty, J.P., Hussein, S., Confalonieri, U., De Wet, N.: Climate change and infectious diseases. Clim. Change Hum. Health Risks Responses 6, 103-137 (2003)

43. Raqib, R., Ahmed, S., Sultana, R., Wagatsuma, Y., Dinesh Mondal, A.M., Hoque, W., Nermell, B., Yunus, M., Roy, S., Persson, L.A., et al.: Effects of in utero arsenic exposure on child immunity and morbidity in rural bangladesh. Toxicol. Lett. 185(3), 197-202 (2009)

44. Rebelo, C., Margheri, A., Bacaër, N.: Persistence in seasonally forced epidemiological models. J. Math. Biol. 64(6), 933-949 (2012)

45. Rodó, X., Pascual, M., Doblas-Reyes, F.J., Gershunov, A., Stone, D.A., Giorgi, F., Hudson, P.J., Kinter, J., Rodríguez-Arias, M.À., Stenseth, N.C., et al.: Climate change and infectious diseases: can we meet the needs for better prediction? Clim. Change 118(3-4), 625-640 (2013) 
46. Ruan, S., Wang, W.: Dynamical behavior of an epidemic model with a nonlinear incidence rate. J. Differ. Equ. 188(1), 135-163 (2003)

47. Schwarzenbach, R.P., Egli, T., Hofstetter, T.B., Von Gunten, U., Wehrli, B.: Global water pollution and human health. Annu. Rev. Environ. Resour. 35, 109-136 (2010)

48. Smith, R.A.: Some applications of Hausdorff dimension inequalities for ordinary differential equations. Proc. R. Soc. Edinb. Sect. A Math. 104(3-4), 235-259 (1986)

49. Tchuenche, J.M., Dube, N., Bhunu, C.P., Smith, R.J., Bauch, C.T.: The impact of media coverage on the transmission dynamics of human influenza. BMC Public Health 11(1), 1 (2011)

50. Tien, J.H., Earn, D.J.D.: Multiple transmission pathways and disease dynamics in a waterborne pathogen model. Bull. Math. Biol. 72(6), 1506-1533 (2010)

51. Van den Driessche, P.: Time delay in epidemic models. IMA Vol. Math. Its Appl. 125, 119-128 (2002)

52. Van den Driessche, P., Watmough, J.: Reproduction numbers and sub-threshold endemic equilibria for compartmental models of disease transmission. Math. Biosci. 180(1), 29-48 (2002)

53. Wang, W.: Global behavior of an SEIRS epidemic model with time delays. Appl. Math. Lett. 15(4), 423-428 (2002)

54. Wang, W.: Backward bifurcation of an epidemic model with treatment. Math. Biosci. 201(1), 58-71 (2006)

55. Yang, H.M., Macoris, M.L.G., Galvani, K.C., Andrighetti, M.T.M., Wanderley, D.M.V.: Assessing the effects of temperature on dengue transmission. Epidemiol. Infect. 137(08), 1179-1187 (2009)

56. Zhang, T., Liu, J., Teng, Z.: Dynamic behavior for a nonautonomous SIRS epidemic model with distributed delays. Appl. Math. Comput. 214(2), 624-631 (2009)

57. Zhang, X., Liu, X.: Backward bifurcation and global dynamics of an sis epidemic model with general incidence rate and treatment. Nonlinear Anal. Real World Appl. 10(2), 565-575 (2009)

58. Zhou, X., Cui, J.: Modeling and stability analysis for a cholera model with vaccination. Math. Methods Appl. Sci. 34(14), 1711-1724 (2011) 